\title{
REDES SOCIAIS COMO ESTRATÉGIA DE PROMOÇÃO DO ALEITAMENTO MATERNO: RELATO DE EXPERIÊNCIA
}

\section{Camila Cioquetta Pereira'; Lisiele Marin Roath'2; Giovana Padoin Brutti3; Leandro da Silva de Medeiros ${ }^{4}$; Giovana Luiza Rossato ${ }^{5}$; Nathália Hoffmann Adames $^{6}$; Dirce Stein Backes ${ }^{7}$}

\section{RESUMO}

Objetiva-se relatar a experiência de acadêmicos do curso de Enfermagem, integrantes do Grupo de Gestantes - GESTAR, na produção mídiatica sobre Aleitamento Materno à luz da temática da Semana Mundial de Aleitamento Materno (SMAM). Métodos: Trata-se de um estudo descritivo, do tipo relato de experiência, desenvolvido por acadêmicos de Enfermagem, integrantes do Grupo de Gestantes GESTAR, da Universidade Franciscana, no mês de agosto de 2021. Resultados: O uso das redes sociais como estratégia de promoção do Aleitamento Materno possibilitou a socialização de postagens criativas e educativas e, ainda, gerou reflexões e ampliou informações em torno dos benefícios do aleitamento materno. Possibilitou-se, ainda, informações de forma clara, segura e interativa às usuárias das diferentes classes sociais. Conclusão: A vivência acadêmica fortaleceu a autonomia e o protagonismo dos estudantes, potencilizou iniciativas interprofissionais e agregou novas competências técnico-científicas.

Palavras-chave: Amamentação; Mídias sociais; Saúde materno-infantil.

Eixo Temático: Atenção Integral e Promoção à Saúde (AIPS).

\footnotetext{
${ }^{1}$ Estudante do Curso de Enfermagem, Universidade Franciscana. Membr do Grupo GESTAR.

E-mail: camila.cioquetta@ufn.edu.br

2 Estudante do Curso de Enfermagem, Universidade Franciscana. Membro do Grupo GESTAR.

E-mail: lisiele.marin@ufn.edu.br

${ }^{3}$ Estudante do Curso de Enfermagem, Universidade Franciscana. Membro do Grupo GESTAR.

E-mail: giovana.pbrutti@ufn.edu.br

${ }^{4}$ Estudante do Curso de Enfermagem, Universidade Franciscana. Bolsista PPSUS/FAPERGS.

E-mail: leandro.medeiros@ufn.edu.br

${ }^{5}$ Estudante do Curso de Enfermagem, Universidade Franciscana. Bolsista PROBIC/UFN.

E-mail: giovana.rossato@ufn.edu.br

${ }^{6}$ Odontológa. Mestre em Saúde Materno Infantil pela Universidade Franciscana - UFN. E-mail: natiadames@gmail.com

${ }_{7}$ Doutora em Enfermagem. Docente do Curso de Enfermagem e Coordenadora do Mestrado

Profissional Saúde Materno Infantil da Universidade Franciscana - UFN.

E-mail: backesdirce@ufn.edu.br
} 


\section{INTRODUÇÃO}

O aleitamento materno é fundamental para o desenvolvimento infantil, principalmente nos dois primeiros anos de vida do bebê. A Organização Mundial da Saúde (OMS), juntamente com o Fundo das Nações Unidas para a infância (UNICEF) e o Ministério da Saúde (MS), recomendam a amamentação na primeira hora de vida, sendo exclusiva nos primeiros seis meses e complementada até 2 anos de idade ou mais (SILVA et al., 2021; MEDEIROS, 2020).

Entre os diversos benefícios do aleitamento materno, destaca-se o leite materno como sendo o único que contém anticorpos e outras substâncias que protegem a criança de infecções comuns, como diarréias, infecções respiratórias, otites e outras (BRASIL, 2019). Além disso, propicia um melhor desenvolvimento do sistema nervoso, forte vínculo com a mãe e menor chance de desenvolverem diabetes, obesidade, hipertensão arterial e vários tipos de câncer na vida adulta.

O Programa Nacional de Incentivo ao Aleitamento Materno (PNIAM), foi instituído no Brasil em 1981 e, a partir desse marco, agregaram-se políticas públicas e ações visando à promoção, proteção e apoio ao aleitamento materno (BARROS et al., 2021). Segundo FONSECA et al. (2021), no cenário mundial, o Brasil destaca-se pelas diversas ações de promoção e incentivo à amamentação criadas no âmbito da saúde pública.

Com o intuito de fortalecer o incentivo ao aleitamento materno, o Ministério da Saúde em parceria com a Sociedade Brasileira de Pediatria (SBP), realiza a Semana Mundial de Aleitamento Materno (SMAM), que ocorre anualmente no mês de Agosto. A SMAM é uma campanha mundial com o objetivo de estimular ações relacionadas com o tema, ainda, teve como temática principal, no ano de 2021 , "Proteger a amamentação: uma responsabilidade de todos" (BRASIL, 2021).

A partir do exposto, objetiva-se relatar a experiência de acadêmicos do curso de Enfermagem, integrantes do Grupo de Gestantes - GESTAR, na produção mídiatica sobre aleitamento materno à luz da temática da Semana Mundial de Aleitamento Materno (SMAM). 


\section{METODOLOGIA}

Trata-se de um estudo descritivo, do tipo relato de experiência, realizado a partir da vivência de acadêmicos integrantes do Grupo de Gestantes - GESTAR, vinculado à Universidade Franciscana - UFN, situada no interior do estado do Rio Grande do Sul.

A experiência consistiu na realização de postagens na rede social Instagram $\AA$, em alusão à Semana Mundial de Aleitamento Materno e Agosto Dourado que visam a promoção, proteção e apoio ao aleitamento materno. As divulgações das postagens ocorreram durante o mês de Agosto de 2021, totalizando 18 publicações. As edições foram realizadas na plataforma Canva e utilizou-se imagens do Google e manuais do Ministérios da Saúde como base teórica.

O grupo GESTAR foi criado no ano de 2016, com o objetivo de mobilizar uma nova cultura obstétrica entre alunos da graduação da área da saúde, residência em enfermagem obstétrica, alunos do mestrado em Saúde Materno Infantil e usuárias da saúde, em geral, gestantes e puérperas. Visa-se, com este grupo, produzir pesquisas e desenvolver as boas práticas em saúde materno-infantil. O grupo encontrava-se quinzenalmente, com base em cronograma de atividades semestrais.

Face à pandemia, o GESTAR reinventou as suas atividades e buscou outras estratégias para promover as boas práticas em saúde materno-infantil, portanto, apropriou-se das redes sociais a fim de transmitir informações através de postagens educativas e demais ações como Grupo de Gestantes Virtuais, Webinar e Simpósios.

Ressalta-se, que o estudo faz parte de uma pesquisa maior intitulado "Qualificação da rede de atenção à saúde materno-infantil na região central do Rio Grande do Sul" que tem parecer favorável do Comitê de Ética em Pesquisa (CEP) sob o número de protocolo: 4.253.922.

\section{RESULTADOS E DISCUSSÕES}

O uso das redes sociais para postagens de posts criativos e educativos gerou reflexões e ampliou informações em torno dos benefícios do aleitamento materno. 
Possibilitou-se informações de forma clara, segura e interativa às usuárias das diferentes classes sociais.

A utilização de novas tecnologias de comunicação e informação tem crescido nos últimos tempos e, devido ao isolamento social, decorrente da pandemia da COVID-19, as redes sociais passaram a ser um instrumento indispensável na propagação de conhecimentos direcionadas à saúde pública, como também, na manutenção da ligação entre as pessoas (CHEN; LERMAN; FERRARA, 2020).

Nesse contexto, foi utilizado pelos estudantes o perfil da rede social Instagram® do GESTAR (@grupo_gestar), para difundir informações e contribuir na construção do conhecimento acerca do aleitamento materno. A página possui 817 seguidores e deteve 357 interações (curtidas, salvamentos e compartilhamentos) com o conteúdo publicado no mês de agosto.

Ao total houveram 18 publicações abordando as dúvidas mais frequentes sobre amamentação, baseadas no material elaborado pelo Ministério da Saúde intitulado "Guia Alimentar para Crianças Brasileiras Menores de 2 Anos". O cronograma de postagens foi discutido entre o grupo e a temática dividida para cada membro, bem como, a paleta de cores foi previamente estabelecida e livre escolha do design.

Desse modo, foi necessário aprender a criar publicações visualmente atrativas e desenvolver habilidades vinculadas à criação do material, o que demandou organização, tempo e estudo para alcançar resultados satisfatórios, uma vez que isso é necessário para que os seguidores do perfil mantenham o interesse em visualizar, curtir e compartilhar as postagens.

A primeira postagem consistiu em abordar a questão relacionada aos questionamentos que muitas gestantes recebem sobre a existência de leite materno fraco (figura 1). A natureza garante que as mulheres produzam leite com calorias, nutrientes, vitaminas, sais minerais, anticorpos, água e todo o resto em quantidades adequadas (BRASIL, 2019), portanto, é correto afirmar que todo leite materno não é fraco e sim totalmente adaptado às necessidades da criança, tornando-se o alimento ideal nos seus primeiros anos de vida. 
Figura 1 - Existe leite fraco?

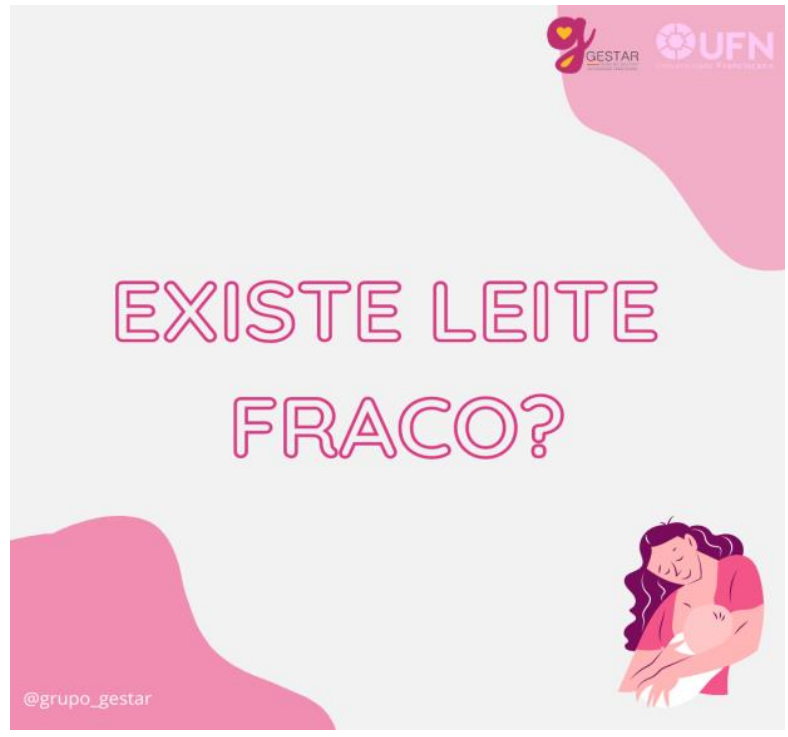

Fonte: Autores.

Nesta sequência, foram publicados também informações como características das mamas e sua influência na amamentação (figura 2), a possibilidade de amamentar um filho adotivo (figura 3), saber se o bebê está recebendo leite materno suficiente (figura 4), saber se o choro do bebê é de fome (figura 5), além de outros assuntos referentes ao aleitamento materno.

Figura 2 - Mamas pequenas produzem pouco leite?

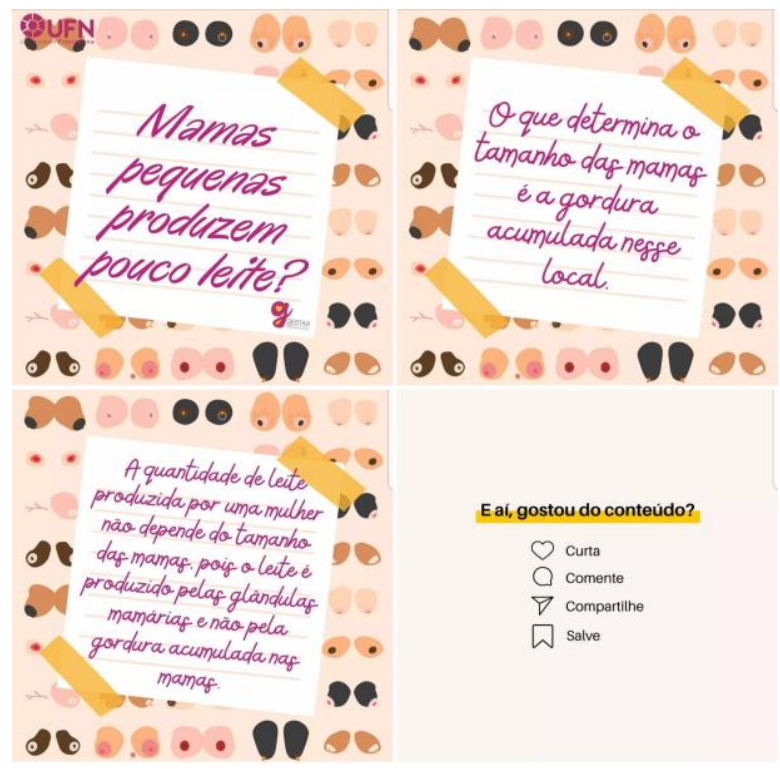

Fonte: Autores. 
EDUCAÇÃO, SAÚDE

ETECNOLOGIA

26 A 28 DE OUTUBRO DE 2021

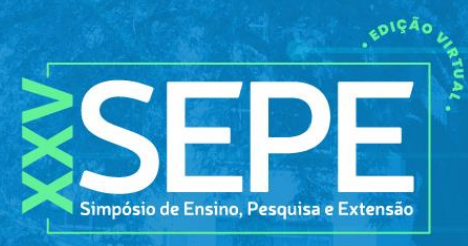

\section{PUFN}

Figura 3 - Uma mulher que adota um filho pode amamentar?

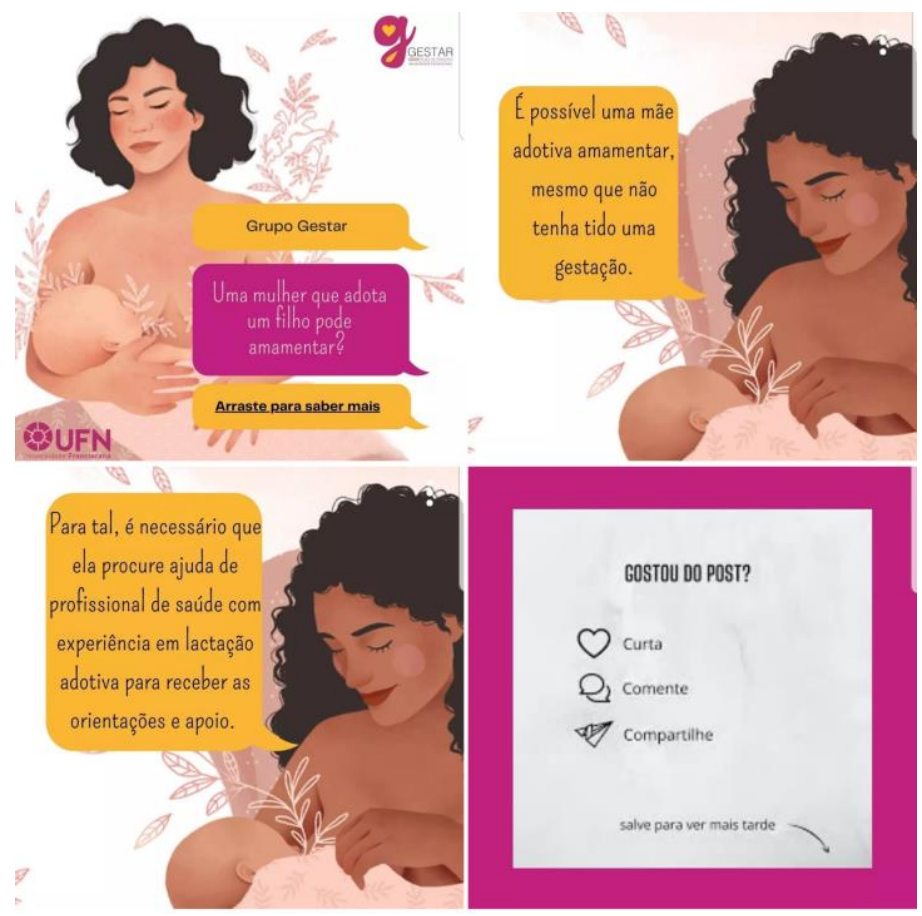

Fonte: Autores.

Figura 4 - Como saber se o bebê está recebendo leite materno suficiente?

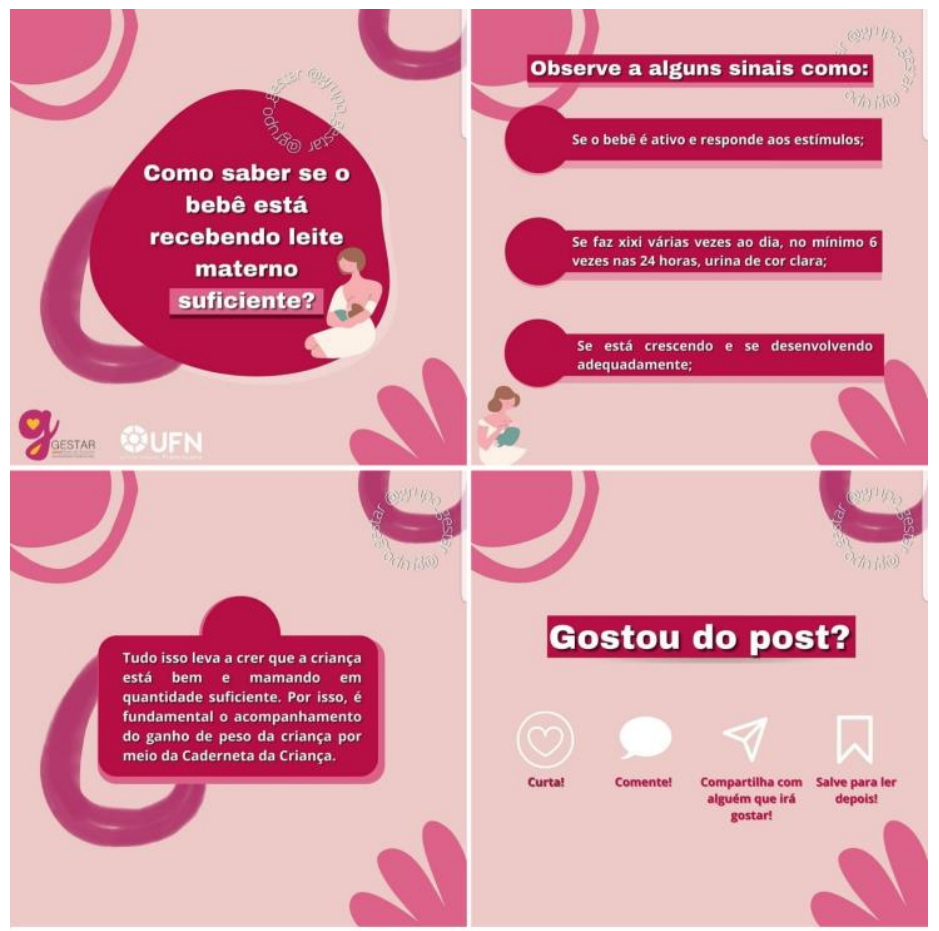

Fonte: Autores. 
Figura 5 - O choro do bebê é de fome?

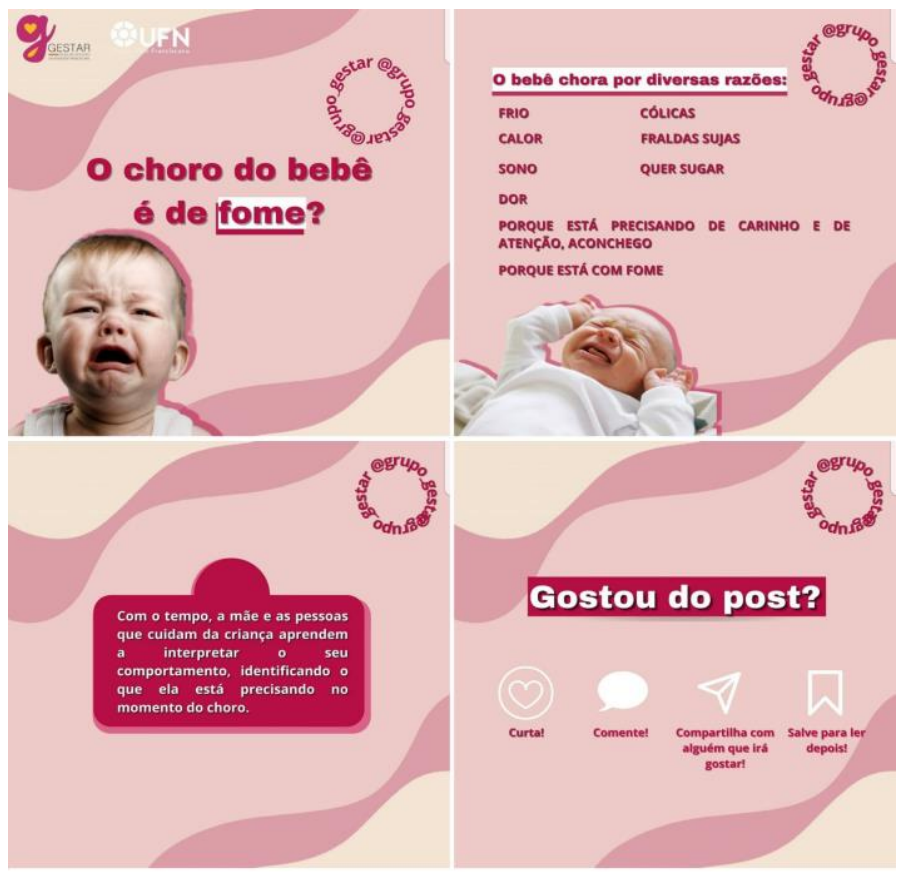

Fonte: Autores.

Além disso, foi desenvolvido diversas temáticas que abordam sobre a importância do aleitamento materno. Ao longo dos tempos, a amamentação vem sendo incentivada e valorizada como importante estratégia de ação para redução da mortalidade infantil pelos órgãos internacionais e colegiados interdisciplinares (MONTESCHIO; GAÍVA; MOREIRA, 2015).

Sobretudo, a lei oㅜ 13.435, de 12 de abril de 2017 institui o Agosto Dourado (BRASIL, 2017) e estipula, no artigo $1^{\circ} \mathrm{em}$ parágrafo único, que "No decorrer do mês de agosto serão intensificadas ações intersetoriais de conscientização e esclarecimento sobre a importância do aleitamento materno". Nessa perspectiva, a responsabilidade de promover, proteger e incentivar a amamentação é compartilhada entre os profissionais de saúde, famílias e toda a sociedade.

Consoante ao que foi exposto, é notória a indispensabilidade desse meio de comunicação para a propagação de conhecimento científico e promoção do aleitamento materno. Além disso, o uso da ferramenta de mídia social Instagram $\circledast$ possibilitou a socialização de informações seguras e de fácil compreensão à população, consolidando o comprometimento e a responsabilidade do grupo. 


\section{CONCLUSÃO}

Conclui-se que a vivência acadêmica fortaleceu a autonomia e o protagonismo dos estudantes, potencilizou iniciativas interprofissionais e agregou novas competências técnico-científicas. A experiência oportunizou ao grupo de estudantes, ainda, um aprofundamento teórico da referida temática, bem como 0 aprimoramento no manuseio das tecnologias. Nesse processo, as redes sociais se constituiram em ferramentas prospectivas de interlocussão com os usuários.

Por fim, a produção midiatica permitiu fortalecer as ações da Semana Mundial de Aleitamento Materno e aos acadêmicos de enfermagem a possibilidade de fortalecer o aprendizado teórico-prático de promoção do aleitamento materno por meio das redes sociais.

\section{REFERÊNCIAS}

BARROS, K. R. S. et al. Perfil epidemiológico e conhecimento de gestantes sobre aleitamento materno em um município do nordeste brasileiro. Arquivos de Ciências da Saúde da UNIPAR, Umuarama, v. 25, n. 1, p, 11-17, jan./abr. 2021.

BRASIL. Lei no 13.435, de 12 de abr. de 2017. Institui o mês de agosto como o Mês do Aleitamento Materno. Brasília, DF, 2017.

BRASIL. Ministério da Saúde. Secretaria de Atenção Primária à Saúde. Departamento de Promoção da Saúde. Guia alimentar para crianças brasileiras menores de 2 anos. Secretaria de Atenção Primária à Saúde. Departamento de Promoção da Saúde. Brasília. 2019.

BRASIL. Rede Global de Bancos de Leite Humano. Semana Mundial de Aleitamento Materno - Agosto Dourado. 2021. Acesso em: 7 Set. 2021. Disponível em: $\quad<$ https://rblh.fiocruz.br/semana-mundial-de-aleitamento-materno-agostodourado-2021>.

CHEN, E.; LERMAN, K.; FERRARA, E. Covid-19:The first public coronavirus twitter dataset. arXiv preprint arXiv:2003.07372, 2020.

FONSECA, R.M.S. et al. O papel do banco de leite humano na promoção da saúde materno infantil: uma revisão sistemática. Ciência \& Saúde Coletiva. 2021. Acesso em: 7 Set. 2021. Disponível em: <DOI: 10.1590/1413-81232020261.24362018>.

MEDEIROS, L. S. Gestação, parto e nascimento na pandemia da COVID-19. Diário de Santa Maria. p. 2, 27 jul. 2020. Disponível em: 


\section{QUPN}

http://https//diariosm.com.br/flip/view/?dataPublicacao=20200918\&publicacaoNome= Jornal\# page/2. Acesso em 19 de Set. 2021.

MONTESCHIO, C.A.C.; GAÍVA, M.A.M.; MOREIRA, M.D.S. The nurse faced with early weaning in child nursing consultations. Rev Bras Enferm., v.68, n.5, p.587-93, 2015.

SILVA, B. S. et al. A amamentação em tempos da COVID-19: uma revisão narrativa. Revista Nursing. 2021. Acesso em: 5 Set. 2021. Disponível em: <http://revistas.mpmcomunicacao.com.br/index.php/revistanursing/article/view/1566/ 1779>. 